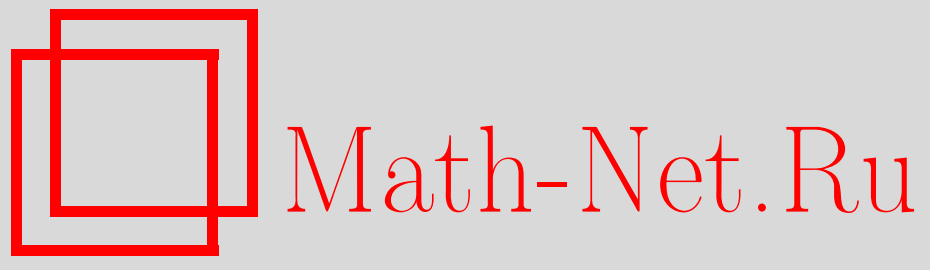

Z. Bao, J. Xie, CLT for linear spectral statistics of Hermitian Wigner matrices with general moment conditions, Теория вероятн. и ее примен., 2015, том 60, выпуск 2, 311-332

DOI: https://doi.org/10.4213/tvp4621

Использование Общероссийского математического портала Math-Net.Ru подразумевает, что вы прочитали и согласны с пользовательским соглашением http://www . mathnet.ru/rus/agreement

Параметры загрузки:

IP : 54.174 .149 .18

26 апреля 2023 г., 13:55:26

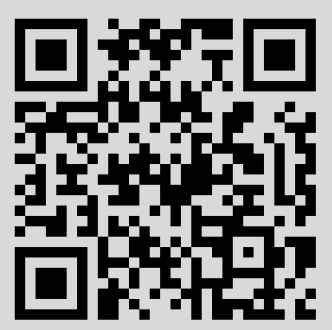




\title{
CLT FOR LINEAR SPECTRAL STATISTICS OF HERMITIAN WIGNER MATRICES WITH GENERAL MOMENT CONDITIONS ${ }^{1)}$
}

\begin{abstract}
Изучается эрмитова матрица Вигнера $W_{n}=\left(x_{i j}\right)_{1 \leqslant i, j \leqslant n}$ с независимыми (с точностью до симметрии) внедиагональными элементами, имеющими нулевое среднее и единичную дисперсию. При некотором условии типа Линдеберга на четвертые моменты элементов матрицы доказана центральная предельная теорема для линейных статистик собственных значений матрицы $W_{n}$. Наш результат распространяет предыдущие результаты подобного рода на более общий случай, когда отсутствует ограничение $\mathbf{E} x_{i j}^{2}=0$ для $1 \leqslant i<j \leqslant n$. Вместо этого мы предполагаем только, что вещественная и мнимая части наддиагональных элементов некоррелированы. Более точно, мы требуем, чтобы математические ожидания $\mathbf{E} x_{i j}^{2}$ были вещественными и однородными для всех $1 \leqslant i<j \leqslant n$. Показано, что предельное нормальное распределение в центральной предельной теореме зависит от параметра $\mathbf{E} x_{i j}^{2} \in[-1,1]$.
\end{abstract}

Ключевые слова и фразы: центральная предельная теорема, линейные спектральные статистики, эрмитовы матрицы Вигнера.

1. Introduction and main result. In this paper, we consider the complex Hermitian Wigner matrix $W_{n}=\left(x_{i j}\right)_{1 \leqslant i, j \leqslant n}$, whose entries $x_{i j}$, $1 \leqslant i \leqslant j \leqslant n$, are independent complex-valued random variables satisfying the following conditions:

(a) for all $i, x_{i i}$ are real and $\mathbf{E} x_{i i}=0, \mathbf{E} x_{i i}^{2}=\omega_{2} \geqslant 0$;

(b) for all $1 \leqslant i<j \leqslant n, x_{i j}:=x_{i j}^{\mathrm{R}}+\sqrt{-1} x_{i j}^{\mathrm{I}}$; here $x_{i j}^{\mathrm{R}}$ and $x_{i j}^{\mathrm{I}}$ are real-valued random variables, satisfying $\mathbf{E} x_{i j}^{\mathrm{R}}=\mathbf{E} x_{i j}^{\mathrm{I}}=0, \mathbf{E}\left(x_{i j}^{\mathrm{R}} x_{i j}^{\mathrm{I}}\right)=0$, $\mathbf{E}\left(x_{i j}^{\mathrm{R}}\right)^{2}=w_{2}^{\mathrm{R}}, \mathbf{E}\left(x_{i j}^{\mathrm{I}}\right)^{2}=w_{2}^{\mathrm{I}}$ such that $w_{2}^{\mathrm{R}}+w_{2}^{\mathrm{I}}=\mathbf{E}\left|x_{i j}\right|^{2}=1 ;$

(c) there exists a positive number $M$ such that $\mathbf{E}\left|x_{i j}\right|^{4}=M$ for all $i \neq j$

(d) for any $\eta>0$,

$$
\frac{1}{\eta^{4} n^{2}} \sum_{i, j=1}^{n} \mathbf{E}\left[\left|x_{i j}\right|^{4} I_{\left\{\left|x_{i j}\right| \geqslant \eta \sqrt{n}\right\}}\right]=o(1) \quad \text { as } n \rightarrow \infty .
$$

${ }^{*}$ Department of Mathematics, Zhejiang University, Hangzhou, P.R. China.

${ }^{* *}$ College of Mathematics and Information Science, Henan University, Kaifeng, P.R. China; e-mail: junshan@henu.edu.cn

1) This work was supported by NSFC (projects 11326173, 11401169), ZJNSF (project R609003), RFDP (project 20100101110001), and HNECF (project 13A110087). 
If we denote the $n$ ordered eigenvalues of $W_{n} / \sqrt{n}$ by $\lambda_{1} \leqslant \lambda_{2} \leqslant \cdots \leqslant \lambda_{n}$, we can define the corresponding empirical spectral distribution of $W_{n} / \sqrt{n}$ by

$$
F_{n}(x)=\frac{1}{n} \sum_{i=1}^{n} I_{\left\{x \geqslant \lambda_{i}\right\}} .
$$

It is well known that almost surely $F_{n}(x)$ converges weakly to the semicircle law $F(x)$ with density function

$$
\rho(x)=\frac{1}{2 \pi} \sqrt{4-x^{2}} I_{\{|x| \leqslant 2\}} .
$$

Moreover, if we define the linear eigenvalue statistics with any bounded continuous test function $f$ as

$$
\mathscr{N}_{n}(f)=\sum_{i=1}^{n} f\left(\lambda_{i}\right),
$$

one has

$$
n^{-1} \mathscr{N}_{n}(f) \stackrel{\mathbf{P}}{\longrightarrow} \int f(x) \rho(x) d x .
$$

This universal result corresponds to the classical law of large numbers for sums of independent random variables. So it is natural to take a step further to study the fluctuations of the linear eigenvalue statistics.

Central limit theorem (CLT) for the fluctuation of $\mathscr{N}_{n}(f)$ has been proven for a lot of random matrix ensembles under different assumptions on either matrix entries or the test function $f$. For Wigner matrices, we refer to Sinaı̆ and Soshnikov [13], Johansson [8], Bai and Yao [4], Lytova and Pastur [16], and Shcherbina [12] for examples.

A remarkable work was done by Bai and Yao [4], where the authors investigated the asymptotics of the spectral empirical process of complex Wigner matrices by using the Stieltjes transform approach and the CLT for martingales. We denote $\mathscr{U}$ to be an open set in the complex plane which contains the interval $[-2,2]$ (the support of $F(x)$ ), and let $\mathscr{A}$ be the set of analytic functions $f: \mathscr{U} \rightarrow \mathbf{C}$. Under the assumptions (a)-(d) and the additional condition $\mathbf{E} x_{i j}^{2}=0$ for $1 \leqslant i<j \leqslant n$, it is proved in [4] that the empirical process $G_{n}:=\left\{G_{n}(f)\right\}$ indexed by $\mathscr{A}$,

$$
G_{n}(f)=n \int_{-\infty}^{+\infty} f(x)\left[F_{n}-F\right](d x), \quad f \in \mathscr{A},
$$

converges weakly in finite dimensions to a Gaussian process. That is, for any $k$ elements $f_{1}, f_{2}, \ldots, f_{k}$ of $\mathscr{A}$, the random vector $\left(G_{n}\left(f_{1}\right), G_{n}\left(f_{2}\right), \ldots, G_{n}\left(f_{k}\right)\right)$ converges weakly to a $k$-dimensional Gaussian distribution. Relying on Bai and Yao's result, under a stronger condition on the entries of the Wigner 
matrices, Bai, Wang and Zhou [3] weakened the condition on test function from analytic to four times continuously differentiable.

Based on [4], we will remove the condition $\mathbf{E} x_{i j}^{2}=0$ in this paper. In this case, $w_{2}^{\mathrm{R}}$ and $w_{2}^{\mathrm{I}}$ may not be equal, i.e., the real part and imaginary part of the upper-diagonal entry can have different variance. Hermitian matrices with «unequal» real and imaginary parts have been discussed comprehensively in the physical literature, see [11] for example. Such a type of random Hermitian matrices is also referred to as the «crossover ensemble», see [7] for further introduction. Our aim is to show that the empirical process $G_{n}=\left\{G_{n}(f)\right\}$ indexed by $\mathscr{A}$ also converges weakly in finite dimensions to a Gaussian process with the general assumption on the parameter $\mathbf{E} x_{i j}^{2}$. Not surprisingly, the mean value function and the covariance function of the limiting process turn out to be parameter-dependent. Particularly, when $\mathbf{E} x_{i j}^{2}=1$ or $\mathbf{E} x_{i j}^{2}=0$, we can recover the results for real or complex Wigner matrices case in [4], respectively. For ease of notation, we denote $\Phi:=\mathbf{E} x_{i j}^{2}=\mathbf{E} \bar{x}_{i j}^{2}=w_{2}^{\mathrm{R}}-w_{2}^{\mathrm{I}}$.

Theorem 1.1. Under the conditions (a)-(d) and $w_{2}^{\mathrm{I}}, w_{2}^{\mathrm{R}}>0$, the spectral empirical process $G_{n}=\left\{G_{n}(f)\right\}$ indexed by the set of analytic functions $\mathscr{A}$ converges weakly in finite dimensions to a Gaussian process $G=\{G(f)\}$ with mean function

$$
\begin{aligned}
\mathbf{E} G(f)= & -\frac{1}{2} \tau_{0}(f)+\left(\omega_{2}-1-\Phi\right) \tau_{2}(f)+\Psi \tau_{4}(f) \\
& +\frac{1-\Phi^{2}}{2 \pi} \int_{-1}^{1} \frac{f(2 t)}{1+\Phi^{2}-2 \Phi T_{2}(t)} \frac{1}{\sqrt{1-t^{2}}} d t
\end{aligned}
$$

and covariance function $c(f, g)=\mathbf{E}[\{G(f)-\mathbf{E} G(f)\}\{G(g)-\mathbf{E} G(g)\}]$ given by

$$
c(f, g)=\omega_{2} \tau_{1}(f) \tau_{1}(g)+2 \Psi \tau_{2}(f) \tau_{2}(g)+\sum_{k=2}^{\infty} k\left(1+\Phi^{k}\right) \tau_{k}(f) \tau_{k}(g)
$$

where the parameter $\Psi$ equals $\mathbf{E}\left|x_{12}\right|^{4}-\Phi^{2}-2$, the function $T_{k}$ is the $k$-th order Chebyshev polynomial satisfying $T_{k}(\cos \theta)=\cos (k \theta)$, and

$$
\tau_{k}(f)=\frac{1}{2 \pi} \int_{-\pi}^{\pi} f(2 \cos \theta) e^{i k \theta} d \theta=\frac{1}{\pi} \int_{-1}^{1} f(2 t) T_{k}(t) \frac{1}{\sqrt{1-t^{2}}} d t .
$$

$\mathrm{R}$ e $\mathrm{m}$ a r k 1.1. When $\omega_{2}^{\mathrm{I}}=0$, it reduces to be the real Wigner matrices case, which has been studied in [4]. Note that $T_{2}(t)=2 t^{2}-1$, thus when $\Phi= \pm 1$, it is not difficult to see that the last term of (1.4) will become indeterminate forms. A separate issue on contour integral with poles is needed for these two cases, here we just exclude them from our theorem for ease of presentation. 
R e m a r k 1.2. By pursuing a Bernstein polynomial approximation method adopted by Bai, Wang and Zhou [3], it is reasonable to assert that our result in this paper can be extended to the case that $f \in C^{4}(\mathscr{U})$ under the conditions (a)-(d) together with the existence of the sixth moments of the entries of $W_{n}$.

$\mathrm{R}$ e $\mathrm{m}$ a $\mathrm{r} \mathrm{k}$ 1.3. A similar result for sample covariance matrices can also be established analogously, we will present it elsewhere. Moreover, for the sample covariance matrices, we do not need the assumption that the real part and imaginary part of the entry to be uncorrelated. We also believe that this assumption can be removed for Wigner matrices. However, it will cause some additional technical difficulty, we do not pursue this direction here.

Our paper is organized as follows. In Section 2 we present the main strategy of the proof and the simplification by truncation. Sections 3 and 4 are devoted to the proof of Theorem 2.1. At the end, in Section 5, we will complete the proof of Theorem 1.1. Some of the background has been laid out in [4], and the main part of the paper are concentrated on the evaluation of some additional terms caused by the lack of the assumption $\Phi=0$.

Throughout the paper, when there is no confusion, we will get rid of the subscript $n$ and the variable $z$ in the notation of matrices. We denote by $A^{*}$ the conjugate transpose of matrix $A$, by $A^{T}$ the transpose of matrix $A$, and by $\operatorname{tr} A$ the trace of $A$. The notation $\|\cdot\|_{\text {op }}$ means the operator norm of a matrix. We use $\sigma\left(\xi_{1}, \ldots, \xi_{k}\right)$ to denote the $\sigma$-field generated by random variables $\xi_{1}, \ldots, \xi_{k}$. The symbol $\stackrel{L_{2}}{\longrightarrow}$ means convergence in the mean square. We write $\xi_{n}=o_{p}\left(a_{n}\right)$ to indicate that $\xi_{n} / a_{n} \rightarrow 0$ in probability as $n \rightarrow \infty$. We also write $C, K$ for positive constants independent of $n$, whose values may change from line to line.

2. Strategy of the proof and simplification by truncation. The basic tool of the proof is the Stieltjes transform, which is frequently used in the random matrix theory. For any real-valued function $H(x)$ with bounded variation, its Stieltjes transform $s_{H}(z)$ is defined on $\{z: z=u+i v, v>0\}$ by

$$
s_{H}(z)=\int_{-\infty}^{\infty} \frac{1}{x-z} d H(x)
$$

Thus by definition the Stieltjes transform of the empirical spectral distribution $F_{n}(x)$ is

$$
s_{n}(z)=\int_{-\infty}^{\infty} \frac{1}{x-z} F_{n}(d x)=\frac{1}{n} \operatorname{tr}\left(\frac{1}{\sqrt{n}} W_{n}-z I_{n}\right)^{-1}
$$


It is well known that as $n$ tends to infinity $s_{n}(z)$ a.s. converges to the Stieltjes transform of the semicircle law,

$$
s(z)=\int \frac{1}{x-z} F(d x) .
$$

Moreover, $s(z)$ satisfies the following equation:

$$
s^{2}(z)+z s(z)+1=0
$$

By the Cauchy integral theorem, we also have the following representation:

$$
\begin{aligned}
G_{n}(f) I_{B_{n}^{c}} & =\frac{1}{2 \pi i} I_{B_{n}^{c}} \int \oint_{\gamma} \frac{f(z)}{z-x} n\left[F_{n}-F\right](d x) d z \\
& =-\frac{1}{2 \pi i} I_{B_{n}^{c}} \oint_{\gamma} f(z) n\left[s_{n}(z)-s(z)\right] d z
\end{aligned}
$$

where the contour $\gamma$ is assumed to be formed by the boundary of the rectangle with vertex $\left( \pm a \pm i \nu_{0}\right)$ with some $a>2,1 \geqslant \nu_{0}>0$, and $a-2$ and $\nu_{0}$ are sufficiently small such that $\gamma \subset \mathscr{U}$. Here $B_{n}=\left\{\max _{i}\left|\lambda_{i}\right| \geqslant 1+a / 2\right\}$. Note that equality (2.2) without the indicator function $I_{B_{n}^{c}}$ may be incorrect when some eigenvalues of $W_{n} / \sqrt{n}$ fall outside the contour $\gamma$. However, the paper [5] reveals that the probability of this event decays rapidly to zero. A routine discussion on this issue leads us to use the following integral instead:

$$
G_{n}(f)=-\frac{1}{2 \pi i} \oint_{\gamma} f(z) n\left[s_{n}(z)-s(z)\right] d z .
$$

For more details, see [2, Chap. 9].

Then by pursuing the Bai-Yao strategy in [4], we can reduce the problem to the convergence of the process $\left\{\xi_{n}(z)\right\}$ indexed by $z \notin[-2,2]$. Here

$$
\xi_{n}(z)=: n\left[s_{n}(z)-s(z)\right], \quad z \notin[-2,2] .
$$

More precisely, if we can get the process $\left\{\xi_{n}(z) ; \mathbf{C}_{0}\right\}$, where $\mathbf{C}_{0}=\{z: \operatorname{Im} z \geqslant$ $\left.v_{0}\right\}$, converged weakly to a Gaussian process, then by the arguments in [4] on the convergence of complex integral on the contour $\gamma$ we can get the conclusion of Theorem 1.1. So at first, we provide the following theorem.

Theorem 2.1. Under the conditions of Theorem 1.1, the process $\left\{\xi_{n}(z) ; \mathbf{C}_{0}\right\}$ converges weakly to a Gaussian process $\left\{\xi(z) ; \mathbf{C}_{0}\right\}$ with mean and covariance functions given in Propositions 3.1 and 4.1 below.

Note that to prove Theorem 1.1, we need to extend the convergence issue of $\left\{\xi_{n}(z)\right\}$ to the region $\mathbf{C} \backslash[-2,2]$ rather than $\mathbf{C}_{0}$. Such an extension has been done in [4], so here we do not repeat the details. The extension of the process allows us to insert the limiting mean and covariance functions 
of $\left\{\xi_{n}(z) ; \mathbf{C}_{0}\right\}$ into the contour integral to get the limit of the mean and variance functions of $\left\{G_{n}(f)\right\}$. Thus throughout the paper, we will restrict our discussion on $\mathbf{C}_{0}$.

In order to control the fluctuation around the extreme eigenvalues, and to apply the concentration Lemma 7.2 in [4], we will also truncate the entries at an appropriate rate without altering the weak limit of the empirical process.

To do this, we select a sequence $\eta_{n}$ such that $\eta_{n} \rightarrow 0$ as $n \rightarrow \infty$. It should be mentioned that the convergence rate of the sequence is assumed to be slow enough. For definiteness, we always assume $\eta_{n}>\ln ^{-1} n$ in the sequel. Setting

$$
\widehat{x}_{i j}=x_{i j} I_{\left\{\left|x_{i j}\right| \leqslant \eta_{n} \sqrt{n}\right\}}, \quad \widetilde{x}_{i j}=\frac{\widehat{x}_{i j}-\mathbf{E} \widehat{x}_{i j}}{\widehat{\sigma}_{i j}},
$$

where

$$
\widehat{\sigma}_{i j}=\sqrt{\mathbf{D} \widehat{x}_{i j}}, \quad 1 \leqslant i<j \leqslant n, \quad \widehat{\sigma}_{i i}=\sqrt{\frac{\mathbf{D} \widehat{x}_{i i}}{\omega_{2}}}, \quad 1 \leqslant i \leqslant n .
$$

We denote by $\widehat{F}_{n}$ and $\widetilde{F}_{n}$ the empirical spectral distributions of the Wigner matrices $n^{-1 / 2}\left(\widehat{x}_{i j}\right)_{1 \leqslant i, j \leqslant n}$ and $n^{-1 / 2}\left(\widetilde{x}_{i j}\right)_{1 \leqslant i, j \leqslant n}$, respectively, and by $\widehat{G}_{n}$ and $\widetilde{G}_{n}$ their corresponding empirical processes. Moreover, we denote by $\tilde{\lambda}_{n j}$ and $\widehat{\lambda}_{n j}$ the $j$-th largest eigenvalues of the matrices $n^{-1 / 2}\left(\widehat{x}_{i j}\right)_{1 \leqslant i, j \leqslant n}$ and $n^{-1 / 2}\left(\widetilde{x}_{i j}\right)_{1 \leqslant i, j \leqslant n}$, respectively. By assumption $(\mathrm{d})$, we can always choose a sequence $\left\{\eta_{n}\right\}$ such that

$$
\begin{aligned}
\mathbf{P}\left(G_{n} \neq \widehat{G}_{n}\right) & \leqslant \mathbf{P}\left(F_{n} \neq \widehat{F}_{n}\right) \leqslant \mathbf{P}\left(\text { for some } i, j, x_{i j} \neq \widehat{x}_{i j}\right) \\
& \leqslant \sum_{i, j} \mathbf{P}\left(\left|x_{i j}\right| \geqslant \eta_{n} \sqrt{n}\right) \leqslant \frac{1}{\eta_{n}^{4} n^{2}} \sum_{i, j} \mathbf{E}\left[\left|x_{i j}\right|^{4} I_{\left\{\left|x_{i j}\right| \geqslant \eta_{n} \sqrt{n}\right\}}\right]=o(1) .
\end{aligned}
$$

And for any analytic function $f$, we have

$$
\begin{aligned}
& \mathbf{E}\left|\widetilde{G}_{n}(f)-\widehat{G}_{n}(f)\right|^{2} \leqslant C \mathbf{E}\left(\sum_{j=1}^{n}\left|\widetilde{\lambda}_{n j}-\widehat{\lambda}_{n j}\right|\right)^{2} \leqslant C n \mathbf{E} \sum_{j=1}^{n}\left|\widetilde{\lambda}_{n j}-\widehat{\lambda}_{n j}\right|^{2} \\
& =C n \mathbf{E} \sum_{i, j}\left|n^{-1 / 2}\left(\widetilde{x}_{i j}-\widehat{x}_{i j}\right)\right|^{2} \\
& \leqslant C\left[\sum_{i, j}\left(\mathbf{E}\left|x_{i j}\right|^{2}\right)\left|1-\widehat{\sigma}_{i j}^{-1}\right|^{2}+\sum_{i, j}\left|\mathbf{E} \widehat{x}_{i j}\right|^{2} \widehat{\sigma}_{i j}^{-2}\right]=o(1)
\end{aligned}
$$

where we used the following estimations which can be found in [4]:

$$
\max _{i, j}\left|1-\widehat{\sigma}_{i j}\right| \rightarrow 0, \quad \sum_{i, j}\left(\mathbf{E}\left|x_{i j}\right|^{2}\right)\left|1-\widehat{\sigma}_{i j}^{-1}\right|^{2} \rightarrow 0 .
$$


From above we know that the truncation does not affect the weak convergence of $G_{n}(f)$. Now we investigate the impact of the truncation on $\mathbf{E} x_{i j}^{2}$.

Note that

$$
\mathbf{E} x_{i j}^{2}-\mathbf{E} \widetilde{x}_{i j}^{2}=\left(1-\frac{1}{\widehat{\sigma}_{i j}^{2}}\right) \mathbf{E} x_{i j}^{2}+\frac{1}{\widehat{\sigma}_{i j}^{2}}\left[\mathbf{E} x_{i j}^{2}-\mathbf{E} \widehat{x}_{i j}^{2}\right]+\frac{1}{\widehat{\sigma}_{i j}^{2}}\left(\mathbf{E} \widehat{x}_{i j}\right)^{2},
$$

it follows from the assumptions (c) and (d) that

$$
\begin{aligned}
\mathbf{E} x_{i j}^{2}-\mathbf{E} \widehat{x}_{i j}^{2} & =\mathbf{E}\left[x_{i j}^{2} I_{\left\{\left|x_{i j}\right| \geqslant \eta_{n} \sqrt{n}\right\}}\right] \leqslant \mathbf{E}\left[\left|x_{i j}\right|^{2} I_{\left\{\left|x_{i j}\right| \geqslant \eta_{n} \sqrt{n}\right\}}\right] \\
& \leqslant \max _{i, j} \frac{1}{\eta_{n}^{2} n} \mathbf{E}\left[\left|x_{i j}\right|^{4} I_{\left\{\left|x_{i j}\right| \geqslant \eta_{n} \sqrt{n}\right\}}\right] \rightarrow 0,
\end{aligned}
$$

and

$$
\left(\mathbf{E} \widehat{x}_{i j}\right)^{2}=\left(\mathbf{E} x_{i j} I_{\left\{\left|x_{i j}\right| \geqslant \eta_{1 n} \sqrt{n}\right\}}\right)^{2} \leqslant \mathbf{E}\left[\left|x_{i j}\right|^{2} I_{\left\{\left|x_{i j}\right| \geqslant \eta_{1 n} \sqrt{n}\right\}}\right] \rightarrow 0 .
$$

Hence, by the above three relations, we have $\mathbf{E} \widetilde{x}_{i j}^{2}=\Phi+o(1)$. Similarly, we can prove $\mathbf{E}\left|x_{i j}\right|^{4}=\mathbf{E}\left|\widetilde{x}_{i j}\right|^{4}+o(1)$. Consequently, we can view that the truncated random variables $\widetilde{x}_{i j}$ have the same mean values and variances as the original random variables. For brevity, in the sequel we recycle the notation $x_{i j}$ to denote the truncated and normalized variables $\widetilde{x}_{i j}$.

3. The mean function of $\xi_{n}(z)$. For convenience, we set $b_{n}(z):=$ $\mathbf{E} \xi_{n}(z)$ below. In this section, we will evaluate the limit of $b_{n}(z)$ for $z \in \mathbf{C}_{0}$. To do this, we first introduce some notation. Let

$$
D(z)=\left(\frac{1}{\sqrt{n}} W_{n}-z I_{n}\right)^{-1}, \quad D_{k}(z)=\left(\frac{1}{\sqrt{n}} W_{n}(k)-z I_{n-1}\right)^{-1}
$$

where $W_{n}(k)$ is the submatrix extracted from $W_{n}$ by deleting its $k$-th row and $k$-th column. We denote by $\alpha_{k}$ the $k$-th column of $W_{n}$ with $x_{k k} / \sqrt{n}$ removed. A crucial technical step of our proof is the following lemma.

Lemma 3.1. Under the assumptions of Theorem 1.1, for complex numbers $z_{1}, z_{2} \in \mathbf{C}_{0}$ we have

$$
\frac{1}{n} \mathbf{E}\left[\operatorname{tr} D\left(z_{1}\right) D^{T}\left(z_{2}\right)\right]=\frac{s\left(z_{1}\right) s\left(z_{2}\right)}{1-\Phi s\left(z_{1}\right) s\left(z_{2}\right)}+o(1) .
$$

P r o o f. For convenience, we use the notation $\widetilde{D}:=\widetilde{D}\left(z_{1}, z_{2}\right)$ to denote $D\left(z_{1}\right) D^{T}\left(z_{2}\right)$ below. Let $E^{\{j, k\}}$ be the $n \times n$ matrix with only one nonzero entry $E_{j k}^{\{j, k\}}=1$. Note that by basic matrix properties, we know

$$
\begin{gathered}
\left|[D(z)]_{j k}\right| \leqslant\|D(z)\|_{\text {op }} \leqslant \frac{1}{\operatorname{Im} z} \\
\left|\left[D\left(z_{1}\right) D^{T}\left(z_{2}\right)\right]_{j k}\right| \leqslant\left\|D\left(z_{1}\right)\right\|_{\text {op }}\left\|D\left(z_{2}\right)\right\|_{\text {op }} \leqslant \frac{1}{\operatorname{Im} z_{1} \operatorname{Im} z_{2}} .
\end{gathered}
$$


Thus the entries of $D\left(z_{1}\right), D\left(z_{2}\right)$, and $\widetilde{D}\left(z_{1}, z_{2}\right)$ are all bounded by constant. We will use these facts without further explanation in the sequel. Now let

$$
W^{\{j, k\}}=W_{n}-\left(1-\frac{\delta_{j k}}{2}\right)\left(x_{j k} E^{\{j, k\}}+\bar{x}_{j k} E^{\{k, j\}}\right),
$$

where $\delta_{j k}$ is the Kronecker delta function. Note $W^{\{j, k\}}$ is derived by replacing $x_{j k}$ and $x_{k j}$ in $W$ with zero, thus $W^{\{j, k\}}$ is independent of $x_{j k}$. Correspondingly we use the notation

$$
D^{\{j, k\}}(z)=\left(\frac{1}{\sqrt{n}} W^{\{j, k\}}-z I_{n}\right)^{-1}, \quad \widetilde{D}^{\{j, k\}}\left(z_{1}, z_{2}\right)=D^{\{j, k\}}\left(z_{1}\right)\left[D^{\{j, k\}}\left(z_{2}\right)\right]^{T} .
$$

The basic formula for invertible $n \times n$ matrices $U, V$,

$$
U^{-1}-V^{-1}=-U^{-1}(U-V) V^{-1},
$$

yields $D(z)=-z^{-1}+z^{-1} n^{-1 / 2} W_{n} D(z)$. Using the well-known fact (see, for example, $[4$, formula $(2.14)])$

$$
n^{-1} \mathbf{E} \operatorname{tr} D(z)-s(z)=O\left(n^{-1}\right)
$$

we obtain

$$
\begin{aligned}
n^{-1} \mathbf{E} \operatorname{tr} \widetilde{D} & =-z_{1}^{-1} n^{-1} \mathbf{E} \operatorname{tr} D\left(z_{2}\right)+z_{1}^{-1} n^{-3 / 2} \mathbf{E} \operatorname{tr} W_{n} \widetilde{D} \\
& =-z_{1}^{-1} s\left(z_{2}\right)+z_{1}^{-1} n^{-3 / 2} \mathbf{E} \operatorname{tr} W_{n} \widetilde{D}+O\left(n^{-1}\right)
\end{aligned}
$$

Thus it suffices to estimate $\mathbf{E} \operatorname{tr} W_{n} \widetilde{D}$. Using the relation (3.3) repeatedly, we get the following representation of $D(z)$ :

$$
\begin{aligned}
D(z)= & D^{\{j, k\}}(z)-\left(1-\frac{\delta_{j k}}{2}\right) D^{\{j, k\}}(z) n^{-1 / 2}\left(x_{j k} E^{\{j, k\}}+\bar{x}_{j k} E^{\{k, j\}}\right) D^{\{j, k\}}(z) \\
& +\left(\left(1-\frac{\delta_{j k}}{2}\right) D^{\{j, k\}}(z) n^{-1 / 2}\left(x_{j k} E^{\{j, k\}}+\bar{x}_{j k} E^{\{k, j\}}\right)\right)^{2} D(z) \\
=: & D^{\{j, k\}}(z)-A^{\{j, k\}}(z)+B^{\{j, k\}}(z),
\end{aligned}
$$

i.e.,

$$
\begin{aligned}
& A^{\{j, k\}}(z):=\left(1-\frac{\delta_{j k}}{2}\right) D^{\{j, k\}}(z) n^{-1 / 2}\left(x_{j k} E^{\{j, k\}}+\bar{x}_{j k} E^{\{k, j\}}\right) D^{\{j, k\}}(z), \\
& B^{\{j, k\}}(z):=\left(\left(1-\frac{\delta_{j k}}{2}\right) D^{\{j, k\}}(z) n^{-1 / 2}\left(x_{j k} E^{\{j, k\}}+\bar{x}_{j k} E^{\{k, j\}}\right)\right)^{2} D(z) .
\end{aligned}
$$

Consequently we can write the following expansion:

$$
\mathbf{E} \operatorname{tr} W_{n} \widetilde{D}=\sum_{j, k} \mathbf{E}\left\{x_{j k} \widetilde{D}_{k j}\right\}
$$




$$
\begin{aligned}
&=\sum_{i=1}^{n} \mathbf{E}\left\{x_{i i}[\right.\left(D^{\{j, k\}}\left(z_{1}\right)-A^{\{j, k\}}\left(z_{1}\right)+B^{\{j, k\}}\left(z_{1}\right)\right) \\
&\left.\left.\times\left(D^{\{j, k\}}\left(z_{2}\right)-A^{\{j, k\}}\left(z_{2}\right)+B^{\{j, k\}}\left(z_{2}\right)\right)^{T}\right]_{i i}\right\} \\
&+ \sum_{j<k} \mathbf{E}\left\{x _ { j k } \left[\left(D^{\{j, k\}}\left(z_{1}\right)-A^{\{j, k\}}\left(z_{1}\right)+B^{\{j, k\}}\left(z_{1}\right)\right)\right.\right. \\
&\left.\left.\times\left(D^{\{j, k\}}\left(z_{2}\right)-A^{\{j, k\}}\left(z_{2}\right)+B^{\{j, k\}}\left(z_{2}\right)\right)^{T}\right]_{k j}\right\} \\
&+\sum_{j<k} \mathbf{E}\left\{\overline { x } _ { j k } \left[\left(D^{\{j, k\}}\left(z_{1}\right)-A^{\{j, k\}}\left(z_{1}\right)+B^{\{j, k\}}\left(z_{1}\right)\right)\right.\right. \\
&\left.\left.\times\left(D^{\{j, k\}}\left(z_{2}\right)-A^{\{j, k\}}\left(z_{2}\right)+B^{\{j, k\}}\left(z_{2}\right)\right)^{T}\right]_{j k}\right\} .
\end{aligned}
$$

For convenience we introduce the notation

$$
R^{\{j, k\}}=\widetilde{D}^{\{j, k\}}+A^{\{j, k\}}\left(z_{1}\right)\left[D^{\{j, k\}}\left(z_{2}\right)\right]^{T}+D^{\{j, k\}}\left(z_{1}\right)\left[A^{\{j, k\}}\left(z_{2}\right)\right]^{T} .
$$

Inserting the representation (3.8) into (3.7), we can write

$$
\begin{aligned}
\mathbf{E} \operatorname{tr} W_{n} \widetilde{D}= & -\sum_{i=1}^{n} \mathbf{E}\left\{x_{i i}\left(A^{\{i, i\}}\left(z_{1}\right)\left[D^{\{i, i\}}\left(z_{2}\right)\right]^{T}\right)_{i i}\right\} \\
& -\sum_{i=1}^{n} \mathbf{E}\left\{x_{i i}\left(D^{\{i, i\}}\left(z_{1}\right)\left[A^{\{i, i\}}\left(z_{2}\right)\right]^{T}\right)_{i i}\right\} \\
& -\sum_{j<k} \mathbf{E}\left\{x_{j k}\left(A^{\{j, k\}}\left(z_{1}\right)\left[D^{\{j, k\}}\left(z_{2}\right)\right]^{T}\right)_{k j}\right\} \\
& -\sum_{j<k} \mathbf{E}\left\{x_{j k}\left(D^{\{j, k\}}\left(z_{1}\right)\left[A^{\{j, k\}}\left(z_{2}\right)\right]^{T}\right)_{k j}\right\} \\
& -\sum_{j<k} \mathbf{E}\left\{\bar{x}_{j k}\left(A^{\{j, k\}}\left(z_{1}\right)\left[D^{\{j, k\}}\left(z_{2}\right)\right]^{T}\right)_{j k}\right\} \\
& -\sum_{j<k} \mathbf{E}\left\{\bar{x}_{j k}\left(D^{\{j, k\}}\left(z_{1}\right)\left[A^{\{j, k\}}\left(z_{2}\right)\right]^{T}\right)_{j k}\right\} \\
& +\sum_{i=1} \mathbf{E}\left\{x_{i i} R_{i i}^{\{i, i\}}\right\}+\sum_{j, k} \mathbf{E}\left\{x_{j k} R_{k j}^{\{j, k\}}\right\} \\
& +\sum_{j<k} \mathbf{E}\left\{\bar{x}_{j k} R_{j k}^{\{j, k\}}\right\} .
\end{aligned}
$$

We now plug the expression of $A^{\{j, k\}}(z)$ in (3.9), by elementary calculation we obtain

$$
n^{-3 / 2} \mathbf{E} \operatorname{tr} W_{n} \widetilde{D}=-\frac{\omega_{2}}{n^{2}} \sum_{i=1}^{n} \mathbf{E}\left\{\widetilde{D}_{i i}^{\{i, i\}} D_{i i}^{\{i, i\}}\left(z_{2}\right)+\widetilde{D}_{i i}^{\{i, i\}} D_{i i}^{\{i, i\}}\left(z_{1}\right)\right\}
$$




$$
\begin{aligned}
& -\frac{\mathbf{E} x_{12}^{2}}{n^{2}} \sum_{j \neq k} \mathbf{E}\left\{\widetilde{D}_{k k}^{\{j, k\}} D_{j j}^{\{j, k\}}\left(z_{2}\right)+\widetilde{D}_{k j}^{\{j, k\}} D_{k j}^{\{j, k\}}\left(z_{1}\right)\right\} \\
& -\frac{1}{n^{2}} \sum_{j \neq k} \mathbf{E}\left\{\widetilde{D}_{k k}^{\{j, k\}} D_{j j}^{\{j, k\}}\left(z_{1}\right)+\widetilde{D}_{j k}^{\{j, k\}} D_{j k}^{\{j, k\}}\left(z_{2}\right)\right\} \\
& +n^{-3 / 2} \sum_{i=1}^{n} \mathbf{E}\left\{x_{i i} R_{i i}^{\{i, i\}}\right\}+n^{-3 / 2} \sum_{j<k} \mathbf{E}\left\{x_{j k} R_{k j}^{\{j, k\}}\right\} \\
& +n^{-3 / 2} \sum_{j<k} \mathbf{E}\left\{\bar{x}_{j k} R_{j k}^{\{j, k\}}\right\} .
\end{aligned}
$$

It is not difficult to show that the last three terms of (3.10) make a total contribution no more than $O\left(n^{-1 / 2}\right)$. To see this, we take $n^{-3 / 2} \sum_{i=1}^{n} \mathbf{E}\left\{x_{i i} R_{i i}^{\{i, i\}}\right\}$ for example. By definition, we can rewrite

$$
B^{\{j, k\}}=\left(\left(1-\frac{\delta_{j k}}{2}\right) D^{\{j, k\}}(z) n^{-1 / 2}\left(x_{j k} E^{\{j, k\}}+\bar{x}_{j k} E^{\{k, j\}}\right)\right)\left(D^{\{j, k\}}-D\right) .
$$

Combining (3.6) with (3.8), it is not difficult to find that

$$
R_{i i}^{\{i, i\}}=\widetilde{D}_{i i}^{\{i, i\}}+C\left(z_{1}, z_{2}\right) n^{-1} x_{i i}^{2}
$$

with some uniformly bounded complex number $C\left(z_{1}, z_{2}\right)$ depending on $z_{1}, z_{2} \in \mathbf{C}_{0}$. Using the fact that $\widetilde{D}_{i i}^{\{i, i\}}$ is independent of $x_{i i}$ we get $\left|\mathbf{E}\left\{x_{i i} R_{i i}^{\{i, i\}}\right\}\right| \leqslant C n^{-1} \mathbf{E}\left|x_{i i}\right|^{3}=O\left(n^{-1}\right)$, and thus

$$
n^{-3 / 2} \sum_{i=1}^{n} \mathbf{E}\left\{x_{i i} R_{i i}^{\{i, i\}}\right\}=O\left(n^{-3 / 2}\right) .
$$

A similar discussion leads to

$$
n^{-3 / 2} \sum_{j<k} \mathbf{E}\left\{x_{j k} R_{k j}^{\{j, k\}}\right\}=O\left(n^{-1 / 2}\right) .
$$

Thus we can discard the last three terms of (3.10).

To evaluate the main term of (3.10), we will replace all $D^{\{j, k\}}$ by $D$ in (3.10) for any $1 \leqslant j, k \leqslant n$ and show such an operation will cause negligible change. That is to say, we need to estimate the differences

$$
\mathbf{E} \widetilde{D}_{k j} D_{k j}\left(z_{1}\right)-\mathbf{E} \widetilde{D}_{k j}^{\{j, k\}} D_{k j}^{\{j, k\}}\left(z_{1}\right), \quad \mathbf{E} \widetilde{D}_{k k} D_{j j}\left(z_{2}\right)-\mathbf{E} \widetilde{D}_{k k}^{\{j, k\}} D_{j j}^{\{j, k\}}\left(z_{2}\right)
$$

for all $1 \leqslant j, k \leqslant n$.

Using relation (3.6), we can get

$$
\begin{aligned}
\widetilde{D}_{k j}^{\{j, k\}} D_{k j}^{\{j, k\}}\left(z_{1}\right)-\widetilde{D}_{k j} D_{k j}\left(z_{1}\right) \\
=\left[\left(D\left(z_{1}\right)+A^{\{j, k\}}\left(z_{1}\right)-B^{\{j, k\}}\left(z_{1}\right)\right)\right. \\
\left.\quad \times\left(D^{T}\left(z_{2}\right)+\left[A^{\{j, k\}}\left(z_{2}\right)\right]^{T}-\left[B^{\{j, k\}}\left(z_{2}\right)\right]^{T}\right)\right]_{k j} \\
\quad \times\left[\left(D\left(z_{1}\right)+A^{\{j, k\}}\left(z_{1}\right)-B^{\{j, k\}}\left(z_{1}\right)\right)\right]_{k j}-\widetilde{D}_{k j} D_{k j}\left(z_{1}\right) .
\end{aligned}
$$


It is elementary to see the $\widetilde{D}_{k j}^{\{j, k\}} D_{k j}^{\{j, k\}}\left(z_{1}\right)$ is a combination of the terms in the form of $\left[O\left(z_{1}\right) P^{T}\left(z_{2}\right)\right]_{k j}\left[Q\left(z_{1}\right)\right]_{k j}$. Here the notation $O, P, Q$ can be $D$, $A^{\{j, k\}}$ or $B^{\{j, k\}}$. The main term $\left[D\left(z_{1}\right) D^{T}\left(z_{2}\right)\right]_{k j}\left[D\left(z_{1}\right)\right]_{k j}$ can be canceled out by $\widetilde{D}_{k j} D_{k j}\left(z_{1}\right)$ by definition of $\widetilde{D}$. To estimate the remaining terms in (3.15), we will only take

$$
\left[A^{\{j, k\}}\left(z_{1}\right) D^{T}\left(z_{2}\right)\right]_{k j}\left[D\left(z_{1}\right)\right]_{k j}
$$

as an example, the other terms are analogous indeed. Hence, we do not provide the detail of the estimation term by term here. To estimate (3.16), we replace $D\left(z_{1}\right)$ and $D\left(z_{2}\right)$ by their decompositions (3.6). Since $x_{j k}$ and $\bar{x}_{j k}$ are independent of $D^{\{j, k\}}(z)$, it is not difficult to derive

$$
\mathbf{E}\left[A^{\{j, k\}}\left(z_{1}\right) D^{T}\left(z_{2}\right)\right]_{k j} D_{k j}\left(z_{1}\right)=\widetilde{C}\left(z_{1}, z_{2}\right) \frac{1}{n} \mathbf{E}\left|x_{j k}\right|^{2}=O\left(n^{-1}\right) .
$$

Here $\widetilde{C}\left(z_{1}, z_{2}\right)$ is a bounded constant depending on $z_{1}, z_{2} \in \mathbf{C}_{0}$. Since the other terms are analogous, we leave them to the reader. We conclude that

$$
\begin{aligned}
\mathbf{E} \widetilde{D}_{k j} D_{k j}\left(z_{1}\right)-\mathbf{E} \widetilde{D}_{k j}^{\{j, k\}} D_{k j}^{\{j, k\}}\left(z_{1}\right) & =O\left(n^{-1}\right), \\
\mathbf{E} \widetilde{D}_{k k} D_{j j}\left(z_{2}\right)-\mathbf{E} \widetilde{D}_{k k}^{\{j, k\}} D_{j j}^{\{j, k\}}\left(z_{2}\right) & =O\left(n^{-1}\right) .
\end{aligned}
$$

Then it follows from (3.10) that

$$
\begin{aligned}
n^{-3 / 2} \mathbf{E} \operatorname{tr} W_{n} \widetilde{D}= & -\frac{\mathbf{E} x_{12}^{2}}{n^{2}}\left\{\mathbf{E} \operatorname{tr} \widetilde{D} \operatorname{tr} D\left(z_{2}\right)+\mathbf{E} \operatorname{tr} \widetilde{D} D^{T}\left(z_{1}\right)\right\} \\
& -\frac{1}{n^{2}}\left\{\mathbf{E} \operatorname{tr} \widetilde{D} \operatorname{tr} D\left(z_{1}\right)+\mathbf{E} \operatorname{tr} \widetilde{D} D\left(z_{2}\right)\right\}+C n^{-1 / 2} \\
= & -\frac{\mathbf{E} x_{12}^{2}}{n^{2}} \mathbf{E} \operatorname{tr} \widetilde{D} \operatorname{tr} D\left(z_{2}\right)-\frac{1}{n^{2}} \mathbf{E} \operatorname{tr} \widetilde{D} \operatorname{tr} D\left(z_{1}\right)+C n^{-1 / 2} \\
= & -\frac{\mathbf{E} x_{12}^{2}}{n} \mathbf{E} \operatorname{tr} \widetilde{D} \mathbf{E} s_{n}\left(z_{2}\right)-\frac{1}{n} \mathbf{E} \operatorname{tr} \widetilde{D} \mathbf{E} s_{n}\left(z_{1}\right)+C n^{-1 / 2} \\
= & -\frac{\mathbf{E} x_{12}^{2}}{n} s\left(z_{2}\right) \mathbf{E} \operatorname{tr} \widetilde{D}-\frac{1}{n} s\left(z_{1}\right) \mathbf{E} \operatorname{tr} \widetilde{D}+C n^{-1 / 2} \\
= & -\frac{\Phi}{n} s\left(z_{2}\right) \mathbf{E} \operatorname{tr} \widetilde{D}-\frac{1}{n} s\left(z_{1}\right) \mathbf{E} \operatorname{tr} \widetilde{D}+o(1) ;
\end{aligned}
$$

in the third equality, we used the fact that $\mathbf{D} s_{n}(z)=O\left(n^{-2}\right)$ (see, e.g., [12, formula (1.13)]) and in the last two steps we used the relation (3.4) and the assumption $\mathbf{E} x_{12}^{2}=\Phi+o(1)$.

Inserting (3.19) into (3.5), we obtain

$$
\frac{1}{n} \mathbf{E} \operatorname{tr} \widetilde{D}=\frac{-s\left(z_{2}\right)}{z_{1}+s\left(z_{1}\right)+\Phi s\left(z_{2}\right)}+o(1)=\frac{s\left(z_{1}\right) s\left(z_{2}\right)}{1-\Phi s\left(z_{1}\right) s\left(z_{2}\right)}+o(1),
$$

where the last equality follows from (2.1). Thus we conclude the proof of Lemma 3.1.

Now we can prove the following proposition. 
Proposition 3.1. For $z \in \mathbf{C}_{0}$, the mean function $b_{n}(z)$ converges uniformly to

$$
b(z)=\left[1+s^{\prime}(z)\right] s^{3}(z)\left[\omega_{2}-1+\Psi s^{2}(z)+\frac{\Phi^{2} s^{2}(z)}{1-\Phi s^{2}(z)}\right]
$$

P r o o f. We introduce the notation

$$
\begin{gathered}
\beta_{k}(z)=-n^{-1 / 2} x_{k k}+z+n^{-1} \alpha_{k}^{*} D_{k} \alpha_{k} \\
\varepsilon_{k}=n^{-1 / 2} x_{k k}-n^{-1} \alpha_{k}^{*} D_{k} \alpha_{k}+\mathbf{E} s_{n}(z), \quad \delta(z)=-\frac{1}{n} \sum_{k=1}^{n} \frac{\varepsilon_{k}}{\beta_{k}\left(z+\mathbf{E} s_{n}(z)\right)} .
\end{gathered}
$$

It follows by [4, equation (2.11)] that the Stieltjes transform $s_{n}(z)$ of the empirical spectral distribution $F_{n}$ can be written as

$$
s_{n}(z)=\frac{1}{n} \operatorname{tr} D=-\frac{1}{z+\mathbf{E} s_{n}(z)}+\delta(z) .
$$

It follows from the relation (2.1) that $\mathbf{E} s_{n}(z)=s(z+\mathbf{E} \delta(z))+\mathbf{E} \delta(z)$. Using the estimation $\mathbf{E}|\delta(z)| \leqslant C n^{-1}$ in [4], we have

$$
b_{n}(z)=\left[1+s^{\prime}(z)\right] n \mathbf{E} \delta(z)(1+o(1))
$$

So we only need to estimate the term $n \mathbf{E} \delta(z)$. Using the identity for any integer $p$,

$$
\frac{1}{u-\epsilon}=\frac{1}{u}\left[1+\frac{\epsilon}{u}+\cdots+\frac{\epsilon^{p}}{u^{p}}+\frac{\epsilon^{p+1}}{u^{p}(u-\epsilon)}\right],
$$

we get

$$
\begin{aligned}
n \delta(z) & =-\sum_{k=1}^{n} \frac{\varepsilon_{k}}{\left[z+\mathbf{E} s_{n}(z)\right]^{2}}-\sum_{k=1}^{n} \frac{\varepsilon_{k}^{2}}{\left[z+\mathbf{E} s_{n}(z)\right]^{3}}-\sum_{k=1}^{n} \frac{\varepsilon_{k}^{3}}{\beta_{k}\left[z+\mathbf{E} s_{n}(z)\right]^{3}} \\
& =: S_{1}+S_{2}+S_{3} .
\end{aligned}
$$

It follows by the results in [4] that

$$
\mathbf{E} S_{1} \rightarrow-s^{3}(z)\left[1+s^{\prime}(z)\right], \quad\left|\mathbf{E} S_{3}\right|=o(1), \quad z+\mathbf{E} s_{n}(z) \rightarrow-s^{-1}(z),
$$

and

$$
\begin{aligned}
\mathbf{E} \varepsilon_{k}^{2} & =\mathbf{E}\left(\varepsilon_{k}-\mathbf{E} \varepsilon_{k}\right)^{2}+O\left(n^{-2}\right) \\
& =\frac{\omega_{2}}{n}+\frac{1}{n^{2}} \mathbf{E}\left[\alpha_{k}^{*} D_{k} \alpha_{k}-\operatorname{tr} D_{k}\right]^{2}+O\left(n^{-2}\right) .
\end{aligned}
$$


Hence it suffices to evaluate $\mathbf{E}\left[\alpha_{k}^{*} D_{k} \alpha_{k}-\operatorname{tr} D_{k}\right]^{2}$. To do this, we denote $D_{k}(z)=:\left(d_{i j}\right)$ and $\alpha_{k}=\left(x_{1 k}, \ldots, x_{k-1, k}, x_{k+1, k}, \ldots, x_{n k}\right)^{*} / \sqrt{n}=:\left(\xi_{i}\right)^{*}$, note that $\alpha_{k}$ and $D_{k}(z)$ are independent, thus

$$
\begin{aligned}
\mathbf{E} & {\left[\alpha_{k}^{*} D_{k} \alpha_{k}-\operatorname{tr} D_{k}\right]^{2}=\mathbf{E}\left[\sum_{i \neq j} \sum_{s \neq t} d_{i j} d_{s t} \bar{\xi}_{i} \xi_{j} \bar{\xi}_{s} \xi_{t}\right]+\mathbf{E}\left[\sum_{i} d_{i i}^{2}\left(\left|\xi_{i}\right|^{2}-1\right)^{2}\right] } \\
= & \mathbf{E} \bar{\xi}_{i}^{2} \mathbf{E} \xi_{j}^{2} \mathbf{E}\left[\sum_{i \neq j} d_{i j}^{2}\right]+\mathbf{E}\left|\bar{\xi}_{i}\right|^{2} \mathbf{E}\left|\xi_{j}\right|^{2} \mathbf{E} \sum_{i \neq j} d_{i j} d_{j i}+\mathbf{E}\left(\left|\xi_{1}\right|^{2}-1\right)^{2} \mathbf{E} \sum_{i} d_{i i}^{2} \\
& =\mathbf{E}\left[\operatorname{tr} D_{k}^{2}\right]+\Phi^{2} \mathbf{E}\left[\operatorname{tr} D_{k} D_{k}^{T}\right]+\Psi \mathbf{E} \sum_{i} d_{i i}^{2}+o(n) .
\end{aligned}
$$

By the results in [4], we know that $n^{-1} \mathbf{E} \operatorname{tr} D_{k}^{2} \rightarrow s^{\prime}(z)$ and $n^{-1} \mathbf{E} \sum_{i} d_{i i}^{2} \rightarrow$ $s^{2}(z)$. As for the term $\mathbf{E}\left[\operatorname{tr} D_{k} D_{k}^{T}\right]$, which is considered to be difficult to deal with in [4] and some related papers, an additional assumption $\Phi=\mathbf{E} x_{i j}^{2}=0$ is made in [4] to avoid the computation. Now applying Lemma 3.1 to

$$
\mathbf{E}\left[\operatorname{tr} D_{k}\left(z_{1}\right) D_{k}^{T}\left(z_{2}\right)\right]
$$

we get $\mathbf{E}\left[\operatorname{tr} D_{k} D_{k}^{T}\right] / n=s^{2}(z) /\left(1-\Phi s^{2}(z)\right)+o(1)$. From relations (3.22)(3.26), we conclude

$$
b_{n}(z) \rightarrow b(z)=\left[1+s^{\prime}(z)\right] s^{3}(z)\left[\omega_{2}-1+\Psi s^{2}(z)+\frac{\Phi^{2} s^{2}(z)}{1-\Phi s^{2}(z)}\right] .
$$

Thus the proof of Proposition 3.1 is completed.

4. Convergence of the process $\left\{\xi_{n}(z)\right\}$. In this section, we establish the weak convergence of the process

$$
\zeta_{n}=\left\{\zeta_{n}(z)=\xi_{n}-\mathbf{E} \xi_{n}, z \in \mathbf{C}_{0}\right\}
$$

The proof includes two parts. First we prove the finite dimensional convergence of $\left\{\zeta_{n}(z), z \in \mathbf{C}_{0}\right\}$, then the proof of tightness is provided. The main part of this section is focused on the finite dimensional convergence.

Write $s_{j}=s\left(z_{j}\right), \mathbf{E}_{k}(\cdot)=\mathbf{E}\left(\cdot \mid \mathscr{F}_{k}\right)$, where $\mathscr{F}_{k}=\sigma\left(x_{i j}, k+1 \leqslant i, j \leqslant n\right)$ for $0 \leqslant k \leqslant n$. Thus $\left(\mathscr{F}_{k}\right)_{k}$ is a filtration decreasing in $k$. Further we set

$$
g_{k}=n^{-1 / 2} x_{k k}-n^{-1}\left(\alpha_{k}^{*} D_{k} \alpha_{k}-\operatorname{tr} D_{k}\right), \quad b_{k}=\frac{\alpha_{k}^{*} D_{k}^{2} \alpha_{k}-\operatorname{tr} D_{k}^{2}}{n\left(z+n^{-1} \operatorname{tr} D_{k}\right)}
$$

Some detailed derivations in [4] tell us that

$$
\zeta_{n}(z)=\sum_{k=1}^{n} \mathbf{E}_{k-1} \phi_{k}(z)+o_{p}(1)
$$


where

$$
\phi_{k}(z)=-\frac{1+n^{-1} \operatorname{tr} D_{k}^{2}}{\left(z+n^{-1} \operatorname{tr} D_{k}\right)^{2}} g_{k}-b_{k}=\frac{\partial}{\partial z} \frac{g_{k}}{z+n^{-1} \operatorname{tr} D_{k}} .
$$

Then the weak convergence of $\left\{\zeta_{n}(z)\right\}$ can be derived from that of $\left\{\sum_{k=1}^{n} \mathbf{E}_{k-1} \phi_{k}(z)\right\}$. The following important lemma will be useful in the proof.

Lemma 4.1. Under the assumptions of Theorem 1.1, for any $1 \leqslant k \leqslant$ $n$, we have

$$
\frac{1}{n^{2}} \sum_{k=1}^{n} \sum_{i, j>k}\left[\mathbf{E}_{k-1} D_{k}\left(z_{1}\right)\right]_{i j}\left[\mathbf{E}_{k-1} D_{k}\left(z_{2}\right)\right]_{i j} \stackrel{\mathbf{P}}{\longrightarrow}-\frac{1}{\Phi}-\frac{1}{\Phi^{2} s_{1} s_{2}} \ln \left(1-\Phi s_{1} s_{2}\right)
$$

for $z_{1}, z_{2} \in \mathbf{C}_{0}$.

P r o o f. To estimate the left hand side of (4.3), we will follow a procedure similar to that performed in [4] when the authors deal with the term

$$
\frac{1}{n^{2}} \sum_{k=1}^{n} \sum_{i, j>k}\left[\mathbf{E}_{k-1} D_{k}\left(z_{1}\right)\right]_{i j}\left[\mathbf{E}_{k-1} D_{k}\left(z_{2}\right)\right]_{j i} .
$$

Let $i, j$ be two indices different from $k$. Similarly to (3.2), we introduce the matrix

$$
W_{k}^{\{i, j\}}=W_{n}(k)-\left(1-\frac{\delta_{i j}}{2}\right)\left(x_{i j} E_{k}^{\{i, j\}}+\bar{x}_{i j} E_{k}^{\{j, i\}}\right), \quad i, j \neq k,
$$

where $E_{k}^{\{i, j\}}$ is obtained by deleting the $k$-th row and $k$-th column of $E^{\{i, j\}}$. Moreover, we set $D_{k}^{\{i, j\}}=\left(n^{-1 / 2} W_{k}^{\{i, j\}}-z I_{n-1}\right)^{-1}$. Then

$$
D_{k}-D_{k}^{\{i, j\}}=-D_{k}^{\{i, j\}} n^{-1 / 2}\left(1-\frac{\delta_{i j}}{2}\right)\left(x_{i j} E_{k}^{\{i, j\}}+\bar{x}_{i j} E_{k}^{\{j, i\}}\right) D_{k} .
$$

By the definition of $D_{k}$, we get

$$
z D_{k}(z)+I_{n-1}=W_{n}(k) D_{k}=\sum_{i, j \neq k} n^{-1 / 2} x_{i j} E_{k}^{\{i, j\}} D_{k}(z) .
$$

This equation together with (4.6) gives us

$$
\begin{aligned}
z_{1} \sum_{i, j>k} & {\left[\mathbf{E}_{k-1} D_{k}\left(z_{1}\right)\right]_{i j}\left[\mathbf{E}_{k-1} D_{k}\left(z_{2}\right)\right]_{i j} } \\
=-\sum_{i>k}\left[\mathbf{E}_{k-1} D_{k}\left(z_{2}\right)\right]_{i i}+n^{-1 / 2} \sum_{i, j>k} & {\left[\mathbf{E}_{k-1} \sum_{m, l \neq k} x_{m l} E_{k}^{\{m, l\}} D_{k}\left(z_{1}\right)\right]_{i j} } \\
\times & {\left[\mathbf{E}_{k-1} D_{k}\left(z_{2}\right)\right]_{i j} }
\end{aligned}
$$




$$
\begin{aligned}
& =-\sum_{i>k}\left[\mathbf{E}_{k-1} D_{k}\left(z_{2}\right)\right]_{i i}+n^{-1 / 2} \sum_{i, j>k}\left[\mathbf{E}_{k-1} \sum_{m, l \neq k} x_{m l} E_{k}^{\{m, l\}} D_{k}^{\{m, l\}}\left(z_{1}\right)\right]_{i j} \\
& \times\left[\mathbf{E}_{k-1} D_{k}\left(z_{2}\right)\right]_{i j} \\
& -n^{-1} \sum_{i, j>k}\left[\mathbf{E}_{k-1} \sum_{m, l \neq k}\left(1-\frac{\delta_{m l}}{2}\right)\left|x_{m l}\right|^{2} E_{k}^{\{m, l\}} D_{k}^{\{m, l\}}\left(z_{1}\right) E_{k}^{\{l, m\}} D_{k}\left(z_{1}\right)\right]_{i j} \\
& \times\left[\mathbf{E}_{k-1} D_{k}\left(z_{2}\right)\right]_{i j} \\
& -n^{-1} \sum_{i, j>k}\left[\mathbf{E}_{k-1} \sum_{m, l \neq k}\left(1-\frac{\delta_{m l}}{2}\right) x_{m l}^{2} E_{k}^{\{m, l\}} D_{k}^{\{m, l\}}\left(z_{1}\right) E_{k}^{\{m, l\}} D_{k}\left(z_{1}\right)\right]_{i j} \\
& \times\left[\mathbf{E}_{k-1} D_{k}\left(z_{2}\right)\right]_{i j} \\
& =-\sum_{i>k}\left[\mathbf{E}_{k-1} D_{k}\left(z_{2}\right)\right]_{i i}+n^{-1 / 2} \sum_{i, j, l>k} x_{i l}\left[\mathbf{E}_{k-1} D_{k}^{\{i, l\}}\left(z_{1}\right)\right]_{l j}\left[\mathbf{E}_{k-1} D_{k}\left(z_{2}\right)\right]_{i j} \\
& -s\left(z_{1}\right) \frac{n-3 / 2}{n} \sum_{i, j>k}\left[\mathbf{E}_{k-1} D_{k}\left(z_{1}\right)\right]_{i j}\left[\mathbf{E}_{k-1} D_{k}\left(z_{2}\right)\right]_{i j}-\sum_{i, j>k ; l \neq k}\left(1-\frac{\delta_{i l}}{2}\right) \\
& \times \mathbf{E}_{k-1}\left[\left(\frac{\left|x_{i l}\right|^{2}-1}{n} s\left(z_{1}\right)+\frac{\left|x_{i l}\right|^{2}}{n}\left(\left[D_{k}^{\{i, l\}}\right]_{l l}-s\left(z_{1}\right)\right)\right)\left[D_{k}\left(z_{1}\right)\right]_{i j}\right] \\
& \times\left[\mathbf{E}_{k-1} D_{k}\left(z_{2}\right)\right]_{i j} \\
& -\frac{1}{n} \sum_{i, j>k ; l \neq k}\left(1-\frac{\delta_{i j}}{2}\right) \mathbf{E}_{k-1}\left\{x_{i l}^{2}\left[D_{k}^{\{i, l\}}\left(z_{1}\right)\right]_{l, i}\left[D_{k}\left(z_{1}\right)\right]_{l, j}\right\}\left[\mathbf{E}_{k-1} D_{k}\left(z_{2}\right)\right]_{i j} \\
& =: R_{1}+R_{2}+R_{*}+R_{3}+R_{4} \text {. }
\end{aligned}
$$

In (4.8), the term $R_{*}$ is propositional to the left-hand side. We claim $R_{3}$ and $R_{4}$ are negligible. Similar terms $\left(T_{3}\right.$ and $\left.T_{4}\right)$ can be found in [4, section 4.4.1], where the authors deal with the convergence of (4.4). The bounds of $R_{3}$ and $R_{4}$ only depend on $\mathbf{E}\left|x_{i j}\right|^{4}$ indeed. So by the same procedures as in [4], we can also get that the terms $R_{3}$ and $R_{4}$ are negligible. The estimates of the corresponding terms in [4] are omitted since the computations are lengthy but elementary (one can find the details in [2]). Here we also do not provide the details for the evaluation of $R_{3}$ and $R_{4}$.

For the term $R_{1}$, an argument in the [4, section 4.4.1] leads to

$$
\frac{1}{n} R_{1}=-\frac{1}{n} \sum_{i>k}\left[\mathbf{E}_{k-1} D_{k}\left(z_{2}\right)\right]_{i i} \stackrel{L_{2}}{\longrightarrow}-\frac{n-k}{n} s\left(z_{2}\right) .
$$

For the term $R_{2}$, we have

$$
\begin{aligned}
R_{2}= & n^{-1 / 2} \sum_{i, j, l>k} x_{i l}\left[\mathbf{E}_{k-1} D_{k}^{\{i, l\}}\left(z_{1}\right)\right]_{l j}\left[\mathbf{E}_{k-1}\left(D_{k}-D_{k}^{\{i, l\}}\right)\left(z_{2}\right)\right]_{i j} \\
& +n^{-1 / 2} \sum_{i, j, l>k} x_{i l}\left[\mathbf{E}_{k-1} D_{k}^{\{i, l\}}\left(z_{1}\right)\right]_{l j}\left[\mathbf{E}_{k-1} D_{k}^{\{i, l\}}\left(z_{2}\right)\right]_{i j} .
\end{aligned}
$$

We claim the second term of the right-hand side of the equation above is 
$o_{p}(n)$. This fact can be obtained by using (9.3.18) in [2], which tells us

$$
n^{-1 / 2} \sum_{j, l>k} x_{i l}\left[\mathbf{E}_{k-1} D_{k}^{\{i, l\}}\left(z_{1}\right)\right]_{l j}\left[\mathbf{E}_{k-1} D_{k}^{\{i, l\}}\left(z_{2}\right)\right]_{i j} \stackrel{L_{2}}{\longrightarrow} 0 \quad \forall 1 \leqslant i \leqslant n .
$$

For the first term of (4.10), we use the fact

$$
\begin{aligned}
D_{k}\left(z_{2}\right)-D_{k}^{\{i, l\}}\left(z_{2}\right)= & -n^{-1 / 2}\left(1-\frac{\delta_{i l}}{2}\right) \\
& \times D_{k}^{\{i, l\}}\left(z_{2}\right)\left(x_{i l} E_{k}^{\{i, l\}}+\bar{x}_{i l} E_{k}^{\{l, i\}}\right) D_{k}\left(z_{2}\right) .
\end{aligned}
$$

Thus we obtain

$$
\begin{aligned}
R_{2}= & -\frac{1}{n} \sum_{i, j, l>k}\left[\mathbf{E}_{k-1} D_{k}^{\{i, l\}}\left(z_{1}\right)\right]_{l j} \\
& \times\left[\mathbf{E}_{k-1}\left(1-\frac{\delta_{i l}}{2}\right) D_{k}^{\{i, l\}}\left(z_{2}\right)\left(x_{i l}^{2} E_{k}^{\{i, l\}}+|x|_{i l}^{2} E_{k}^{\{l, i\}}\right) D_{k}\left(z_{2}\right)\right]_{i j}+o_{p}(n) \\
= & -\frac{1}{n} \sum_{i, j, l>k}\left(1-\frac{\delta_{i l}}{2}\right) x_{i l}^{2}\left[\mathbf{E}_{k-1} D_{k}^{\{i, l\}}\left(z_{1}\right)\right]_{l j}\left[\mathbf{E}_{k-1} D_{k}^{\{i, l\}}\left(z_{2}\right)\right]_{i i}\left[D_{k}\left(z_{2}\right)\right]_{l j} \\
& -\frac{1}{n} \sum_{i, j, l>k}\left(1-\frac{\delta_{i l}}{2}\right)\left|x_{i l}\right|^{2}\left[\mathbf{E}_{k-1} D_{k}^{\{i, l\}}\left(z_{1}\right)\right]_{l j}\left[\mathbf{E}_{k-1} D_{k}^{\{i, l\}}\left(z_{2}\right)\right]_{i l}\left[D_{k}\left(z_{2}\right)\right]_{i j} \\
& +o_{p}(n) .
\end{aligned}
$$

Then we use the fact

$$
\left.\mathbf{E}\left|\frac{1}{n} \sum_{i, j, l>k}\right| x_{i l}\right|^{2}\left[\mathbf{E}_{k-1} D_{k}^{\{i, l\}}\left(z_{1}\right)\right]_{l j}\left[\mathbf{E}_{k-1} D_{k}^{\{i, l\}}\left(z_{2}\right)\right]_{i l}\left[D_{k}\left(z_{2}\right)\right]_{i j} \mid=O\left(n^{1 / 2}\right),
$$

which is proved in [4, section 4.4.2]. Thus we can discard the second term in (4.12) to get

$$
\begin{aligned}
R_{2}= & -\frac{1}{n} \sum_{i, j, l>k}\left(1-\frac{\delta_{i l}}{2}\right) x_{i l}^{2}\left[\mathbf{E}_{k-1} D_{k}^{\{i, l\}}\left(z_{1}\right)\right]_{l j}\left[\mathbf{E}_{k-1} D_{k}^{\{i, l\}}\left(z_{2}\right)\right]_{i i}\left[D_{k}\left(z_{2}\right)\right]_{l j} \\
& +o_{p}(n) \\
= & -\frac{1}{n} \sum_{i, j, l>k}\left(1-\frac{\delta_{i l}}{2}\right) x_{i l}^{2}\left[\mathbf{E}_{k-1} D_{k}^{\{i, l\}}\left(z_{1}\right)\right]_{l j}\left[\mathbf{E}_{k-1} D_{k}^{\{i, l\}}\left(z_{2}\right)\right]_{i i}\left[D_{k}^{\{i, l\}}\left(z_{2}\right)\right]_{l j} \\
& +o_{p}(n) .
\end{aligned}
$$

Here at the last step, we replaced $D_{k}\left(z_{2}\right)$ by $D_{k}^{\{i, l\}}\left(z_{2}\right)$. Such a replacement is harmless, which can be proved by using the decomposition (4.11) and an argument similar to the estimate (3.17). Furthermore, by the fact (see $[2$, Lemma 9.9]) $\left[D_{k}^{\{i, l\}}\left(z_{2}\right)\right]_{i i}-s\left(z_{2}\right) \stackrel{L_{2}}{\longrightarrow} 0$, one can write $R_{2}=-\frac{1}{n} s\left(z_{2}\right) \sum_{i, j, l>k}\left(1-\frac{\delta_{i l}}{2}\right) x_{i l}^{2}\left[\mathbf{E}_{k-1} D_{k}^{\{i, l\}}\left(z_{1}\right)\right]_{l j}\left[\mathbf{E}_{k-1} D_{k}^{\{i, l\}}\left(z_{2}\right)\right]_{l j}+o_{p}(n)$. 
We claim that

$$
R_{2}=-\frac{1}{n} \Phi s\left(z_{2}\right) \sum_{i, j, l>k}\left(1-\frac{\delta_{i l}}{2}\right)\left[\mathbf{E}_{k-1} D_{k}^{\{i, l\}}\left(z_{1}\right)\right]_{l j}\left[\mathbf{E}_{k-1} D_{k}^{\{i, l\}}\left(z_{2}\right)\right]_{l j}+o_{p}(n) .
$$

This step is a consequence of

$$
\begin{aligned}
-\frac{1}{n} s\left(z_{2}\right) \sum_{j, l>k}\left(1-\frac{\delta_{i l}}{2}\right) \mathbf{E} \mid\left[x_{i l}^{2}\right. & -\Phi]\left.\left[\mathbf{E}_{k-1} D_{k}^{\{i, l\}}\left(z_{1}\right)\right]_{l j}\left[\mathbf{E}_{k-1} D_{k}^{\{i, l\}}\left(z_{2}\right)\right]_{l j}\right|^{2} \\
& =o_{L_{2}}(1)
\end{aligned}
$$

Note that this estimate coincides with $(9.3 .20)$ in $[2]$ if one replaces $\left[D_{k}^{\{i, l\}}\right]_{l j}$ by $\left[\left(D_{k}^{\{i, l\}) T}\right]_{j l}\right.$ in (4.13). Thus we get

$$
\begin{aligned}
R_{2} & =-\Phi \frac{n-k}{n} s\left(z_{2}\right) \sum_{j, l>k}\left[\mathbf{E}_{k-1} D_{k}^{\{i, l\}}\left(z_{1}\right)\right]_{l j}\left[\mathbf{E}_{k-1} D_{k}^{\{i, l\}}\left(z_{2}\right)\right]_{l j}+o_{p}(n) \\
& =-\Phi \frac{n-k}{n} s\left(z_{2}\right) \sum_{j, l>k}\left[\mathbf{E}_{k-1} D_{k}\left(z_{1}\right)\right]_{l j}\left[\mathbf{E}_{k-1} D_{k}\left(z_{2}\right)\right]_{l j}+o_{p}(n) .
\end{aligned}
$$

Here in the last equality we used the fact

$$
\begin{gathered}
\sum_{l>k}\left[\mathbf{E}_{k-1} D_{k}\left(z_{1}\right)\right]_{l j}\left[\mathbf{E}_{k-1} D_{k}\left(z_{2}\right)\right]_{l j}-\sum_{l>k}\left[\mathbf{E}_{k-1} D_{k}^{\{i, l\}}\left(z_{1}\right)\right]_{l j}\left[\mathbf{E}_{k-1} D_{k}^{\{i, l\}}\left(z_{2}\right)\right]_{l j} \\
=o_{p}(1) .
\end{gathered}
$$

To see (4.15), we give the estimation of the difference caused by the replacement of $D_{k}^{\{i, l\}}\left(z_{2}\right)$ by $D_{k}\left(z_{2}\right)$ as an illustration. Note that by (4.11), we have

$$
\begin{aligned}
& \mathbf{E}\left|\sum_{l>k}\left[\mathbf{E}_{k-1} D_{k}^{\{i, l\}}\left(z_{1}\right)\right]_{l j} \mathbf{E}_{k-1}\left(\left[D_{k}^{\{i, l\}}\left(z_{2}\right)\right]_{l j}-\left[D_{k}\left(z_{2}\right)\right]_{l j}\right)\right|^{2} \\
& =\frac{1}{n} \mathbf{E} \mid \sum_{l>k}\left[\mathbf{E}_{k-1} D_{k}^{\{i, l\}}\left(z_{1}\right)\right]_{l j} \\
& \quad \quad \times\left.\mathbf{E}_{k-1}\left(\left(1-\frac{\delta_{i l}}{2}\right) D_{k}^{\{i, l\}}\left(z_{2}\right)\left(x_{i j} E_{k}^{\{i, l\}}+\bar{x}_{i l} E_{k}^{\{i, l\}}\right) D_{k}\left(z_{2}\right)\right)\right|^{2} \\
& \leqslant \frac{1}{n} C\left(z_{1}, z_{2}\right) \mathbf{E}\left|x_{i l}\right|^{2}=O\left(\frac{1}{n}\right)
\end{aligned}
$$

where $C\left(z_{1}, z_{2}\right)$ is some positive constant depending on $z_{1}, z_{2}$. Consequently we see that the difference caused by such a replacement is $o_{p}(1)$.

By (4.8), (4.9), and (4.14), we obtain

$$
\begin{aligned}
& z_{1} \sum_{i, j>k}\left[\mathbf{E}_{k-1} D_{k}\left(z_{1}\right)\right]_{i j}\left[\mathbf{E}_{k-1} D_{k}\left(z_{2}\right)\right]_{i j} \\
& =-s\left(z_{1}\right)\left[\mathbf{E}_{k-1} D_{k}\left(z_{1}\right)\right]_{i j}\left[\mathbf{E}_{k-1} D_{k}\left(z_{2}\right)\right]_{i j}-(n-k) s\left(z_{2}\right) \\
& \quad-\Phi \frac{n-k}{n} s\left(z_{2}\right) \sum_{i, j>k}\left[\mathbf{E}_{k-1} D_{k}\left(z_{1}\right)\right]_{i j}\left[\mathbf{E}_{k-1} D_{k}\left(z_{2}\right)\right]_{i j}+\Delta_{k} .
\end{aligned}
$$


Here $\Delta_{k}=o_{p}(n)$ uniformly in $k=1, \ldots, n$. By (4.16), one has

$$
\sum_{i, j>k}\left[\mathbf{E}_{k-1} D_{k}\left(z_{1}\right)\right]_{i j}\left[\mathbf{E}_{k-1} D_{k}\left(z_{2}\right)\right]_{i j}=\frac{-(n-k) s\left(z_{2}\right)+\Delta_{k}}{z_{1}+s\left(z_{1}\right)+\Phi \frac{n-k}{n} s\left(z_{2}\right)} .
$$

Moreover, by the fact $z_{1}+s\left(z_{1}\right)=-1 / s\left(z_{1}\right)$, we finally get

$$
\sum_{i, j>k}\left[\mathbf{E}_{k-1} D_{k}\left(z_{1}\right)\right]_{i j}\left[\mathbf{E}_{k-1} D_{k}\left(z_{2}\right)\right]_{i j}=\frac{(n-k) s\left(z_{1}\right) s\left(z_{2}\right)-\Delta_{k} s\left(z_{1}\right)}{1-\Phi \frac{n-k}{n} s\left(z_{1}\right) s\left(z_{2}\right)} .
$$

Consequently, when $n \rightarrow \infty$, we have

$$
\begin{aligned}
& \frac{1}{n^{2}} \sum_{k=1}^{n} \sum_{i, j>k}\left[\mathbf{E}_{k-1} D_{k}\left(z_{1}\right)\right]_{i j}\left[\mathbf{E}_{k-1} D_{k}\left(z_{2}\right)\right]_{i j} \\
& \quad=\frac{1}{n} \sum_{k=1}^{n} \frac{\frac{n-k}{n} s\left(z_{1}\right) s\left(z_{2}\right)}{1-\Phi \frac{n-k}{n} s\left(z_{1}\right) s\left(z_{2}\right)}+o_{p}(1) \\
& \quad \stackrel{\mathbf{P}}{\longrightarrow} s_{1} s_{2} \int_{0}^{1} \frac{t}{1-\Phi t s_{1} s_{2}} d t=-\frac{1}{\Phi}-\frac{1}{\Phi^{2} s_{1} s_{2}} \ln \left(1-\Phi s_{1} s_{2}\right) .
\end{aligned}
$$

Thus we get the conclusion. Lemma 4.1 is proved.

Proposition 4.1. Under the assumptions (a)-(d), for any set $\left\{z_{s}, s=\right.$ $1, \ldots, p\}$ of $p$ points in $\mathbf{C}_{0}$, the random vector $\left(\zeta_{n}\left(z_{1}\right), \ldots, \zeta_{n}\left(z_{s}\right)\right)$ converges weakly in finite dimensions to a p-dimensional zero mean Gaussian process with covariance matrix given by

$$
\begin{aligned}
\Gamma\left(z_{i}, z_{j}\right)=\frac{\partial^{2}}{\partial z_{i} \partial z_{j}}[ & \left(\omega_{2}-1-\Phi\right) s_{i} s_{j}+\frac{1}{2} \Psi\left(s_{i} s_{j}\right)^{2} \\
& \left.\quad-\ln \left(1-s_{i} s_{j}\right)-\ln \left(1-\Phi s_{i} s_{j}\right)\right] \\
= & s_{i}^{\prime} s_{j}^{\prime}\left[\omega_{2}-1-\Phi+2 \Psi s_{i} s_{j}+\frac{1}{\left(1-s_{i} s_{j}\right)^{2}}+\frac{\Phi}{\left(1-\Phi s_{i} s_{j}\right)^{2}}\right] .
\end{aligned}
$$

$\mathrm{P}$ r o o f. The proof of this proposition will employ the central limit theorem for martingales. We denote

$$
\Gamma_{n}\left(z_{i}, z_{j}\right)=\sum_{k=1}^{n} \mathbf{E}_{k}\left[\mathbf{E}_{k-1} \phi_{k}\left(z_{i}\right) \mathbf{E}_{k-1} \phi_{k}\left(z_{j}\right)\right],
$$

then we need to check the following two conditions.

C o n d it i o n 4.1 (conditional covariance). $\Gamma_{n}$ converges in probability to the matrix $\Gamma$.

C o n d i t i o n 4.2 (conditional Lyapunov condition). For some $q>2$,

$$
\sum_{k=1}^{n} \mathbf{E}_{k}\left[\left\|\mathbf{E}_{k-1} \phi_{k}\right\|^{q}\right] \stackrel{\mathbf{P}}{\longrightarrow} 0 .
$$


Below we will verify these two conditions. For Condition 4.1, a corresponding result in [4] reveals that

$$
\Gamma_{n}\left(z_{1}, z_{2}\right)=\frac{\partial^{2}}{\partial z_{1} \partial z_{2}}\left[s\left(z_{1}\right) s\left(z_{2}\right) \widetilde{\Gamma}_{n}\left(z_{1}, z_{2}\right)+o_{p}(1)\right]
$$

where

$$
\begin{array}{r}
\widetilde{\Gamma}_{n}\left(z_{1}, z_{2}\right)=\sum_{k=1}^{n} \mathbf{E}_{k}\left[\frac{x_{k k}^{2}}{n}+\frac{1}{n^{2}}\right. \\
\mathbf{E}_{k-1}\left(\alpha_{k}^{*} D_{k} \alpha_{k}-\operatorname{tr} D_{k}\right)\left(z_{1}\right) \\
\left.\times \mathbf{E}_{k-1}\left(\alpha_{k}^{*} D_{k} \alpha_{k}-\operatorname{tr} D_{k}\right)\left(z_{2}\right)\right] .
\end{array}
$$

By the same arguments as in (3.26), we have

$$
\begin{aligned}
\widetilde{\Gamma}_{n}\left(z_{1}, z_{2}\right)= & \omega_{2}+\frac{1}{n^{2}} \sum_{k=1}^{n} \sum_{i, j>k}\left[\mathbf{E}_{k-1} D_{k}\left(z_{1}\right)\right]_{i j}\left[\mathbf{E}_{k-1} D_{k}\left(z_{2}\right)\right]_{j i} \\
& +\frac{\Psi}{n^{2}} \sum_{k=1}^{n} \sum_{i>k}\left[\mathbf{E}_{k-1} D_{k}\left(z_{1}\right)\right]_{i i}\left[\mathbf{E}_{k-1} D_{k}\left(z_{2}\right)\right]_{i i} \\
& +\frac{\Phi^{2}}{n^{2}} \sum_{k=1}^{n} \sum_{i, j>k}\left[\mathbf{E}_{k-1} D_{k}\left(z_{1}\right)\right]_{i j}\left[\mathbf{E}_{k-1} D_{k}\left(z_{2}\right)\right]_{i j} \\
=: & \omega_{2}+T_{1 n}+T_{2 n}+T_{3 n} .
\end{aligned}
$$

From [4], we know that

$$
T_{1 n} \stackrel{\mathbf{P}}{\longrightarrow}-1-\left(s_{1} s_{2}\right)^{-1} \ln \left(1-s_{1} s_{2}\right), \quad T_{2 n} \stackrel{L_{2}}{\longrightarrow} \frac{1}{2} \Psi s_{1} s_{2} .
$$

The term $T_{3 n}$ has been considered in Lemma 4.1. Then it follows from (4.20) that Condition 4.1 is satisfied.

Condition 4.2 is proved in [4] for $q=4$. Thus Proposition 4.1 holds.

$\mathrm{Up}$ to now, we have proven the finite dimensional convergence of $\left\{\zeta_{n}(z), z \in \mathbf{C}_{0}\right\}$. Moreover, the proof of the tightness property is the same as that of Bai and Yao [4], which does not depend on their additional condition $\mathbf{E} x_{i j}^{2}=0$, so we omit it here. Theorem 2.1 is proved.

5. Proof of Theorem 1.1. Indeed, by the arguments in [4, section $2.1,2.3,3.1$, and 4.3 , if the process $\left\{\xi_{n}(z) ; \mathbf{C}_{0}\right\}$ converges weakly to a Gaussian process $\left\{\xi(z) ; \mathbf{C}_{0}\right\}$ with the mean and covariance functions given by Propositions 3.1 and 4.1, one can get that the empirical process $G_{n}=\left\{G_{n}(f)\right\}, f \in \mathscr{A}$, converges weakly in finite dimensions to a Gaussian process $G=\{G(f)\}, f \in \mathscr{A}$.

The remaining part of the proof is devoted to calculate the exact values of the mean and covariance functions of the limiting process $\{G(f)\}$. 
Using (2.2), we have

$$
\begin{aligned}
& \mathbf{E} G_{n}(f)=-\frac{1}{2 \pi i} \oint_{\gamma} f(z) \mathbf{E} \xi_{n}(z) d z \\
& \rightarrow \mathbf{E} G(f)=-\frac{1}{2 \pi i} \oint_{\gamma} f(z)\left[1+s^{\prime}(z)\right] s^{3}(z) \\
& \qquad {\left[\omega_{2}-1+\Psi s^{2}(z)+\frac{\Phi^{2} s^{2}(z)}{1-\Phi s^{2}(z)}\right] d z }
\end{aligned}
$$

and

$$
\begin{aligned}
\operatorname{Cov} & \left(G_{n}(f), G_{n}(g)\right)=-\frac{1}{4 \pi^{2}} \oint_{\gamma_{1}} \oint_{\gamma_{2}} f\left(z_{1}\right) g\left(z_{2}\right) \operatorname{Cov}\left(\xi_{n}\left(z_{1}\right), \xi_{n}\left(z_{2}\right)\right) d z_{1} d z_{2} \\
\rightarrow & c(f, g)=-\frac{1}{4 \pi^{2}} \oint_{\gamma_{1}} \oint_{\gamma_{2}} f\left(z_{1}\right) g\left(z_{2}\right) s_{1}^{\prime} s_{2}^{\prime} \\
& \times\left[\omega_{2}-1-\Phi+2 \Psi s_{1} s_{2}+\frac{1}{\left(1-s_{1} s_{2}\right)^{2}}+\frac{\Phi}{\left(1-\Phi s_{1} s_{2}\right)^{2}}\right] d z_{1} d z_{2}
\end{aligned}
$$

where $\gamma_{j}, j=1,2$, are two disjoint contours with vertex $\pm\left(2+\varepsilon_{j}\right) \pm i v_{j}$, both $\varepsilon_{j}$ and $v_{j}$ are very small positive numbers such that the contours are contained in $\mathscr{U}$.

Note that the assumption $w_{2}^{\mathrm{I}}, w_{2}^{\mathrm{R}}>0$ implies $|\Phi|<1$. By the results in [4, section 5.1], we know

$$
-\frac{1}{2 \pi i} \oint_{\gamma} f(z)\left[1+s^{\prime}(z)\right] s^{3}(z)\left[\omega_{2}-1+\Psi s^{2}(z)\right] d z=\left(\omega_{2}-1\right) \tau_{2}(f)+\Psi \tau_{4}(f)
$$

and

$$
\begin{gathered}
-\frac{1}{4 \pi^{2}} \oint_{\gamma_{1}} \oint_{\gamma_{2}} f\left(z_{1}\right) g\left(z_{2}\right) s_{1}^{\prime} s_{2}^{\prime}\left[\omega_{2}-1-\Phi+2 \Psi s_{1} s_{2}+\frac{1}{\left(1-s_{1} s_{2}\right)^{2}}\right] d z_{1} d z_{2} \\
=\left(\omega_{2}-\Phi-1\right) \tau_{1}(f) \tau_{1}(g)+2 \Psi \tau_{2}(f) \tau_{2}(g)+\sum_{\ell=1}^{\infty} \ell \tau_{\ell}(f) \tau_{\ell}(g)
\end{gathered}
$$

where $\tau_{k}(f)$ are defined in (1.6).

At last, we still need to estimate the integral values of the additional terms in (5.1) and (5.2) caused by $\Phi \neq 0$. To this end, select $\rho$ to be $<1$ but so close to 1 that the contour $\gamma^{\prime}=\left\{z=-\left(\rho e^{i \theta}+\rho^{-1} e^{-i \theta}\right): 0 \leqslant \theta<2 \pi\right\}$ is completely contained in the analytic region of $f$. We will use the fact that when $z$ runs a cycle along $\gamma^{\prime}$ counterclockwise, $s$ runs a cycle along the circle $|s|=\rho$ counterclockwise because $z=-\left(s+s^{-1}\right)$. By the Cauchy theorem, we have

$$
\oint_{\gamma} f(z)\left[1+s^{\prime}(z)\right] \frac{s^{5}(z)}{1-\Phi s^{2}(z)} d z=\oint_{\gamma^{\prime}} f(z)\left[1+s^{\prime}(z)\right] \frac{s^{5}(z)}{1-\Phi s^{2}(z)} d z .
$$


Changing variable $z$ to $s$ and noticing that $s^{\prime}=s^{2} /\left(1-s^{2}\right)$ yield

$$
\oint_{\gamma^{\prime}} f(z)\left[1+s^{\prime}(z)\right] \frac{s^{5}(z)}{1-\Phi s^{2}(z)} d z=\oint_{|s|=\rho} f\left(-s-s^{-1}\right) \frac{s^{3}}{1-\Phi s^{2}} d s .
$$

Thus, using the facts that $\rho<1$ and there is no pole inside the circle $|s|=1$, we obtain

$$
\oint_{|s|=\rho} f\left(-s-s^{-1}\right) \frac{s^{3}}{1-\Phi s^{2}} d s=\oint_{|s|=1} f\left(-s-s^{-1}\right) \frac{s^{3}}{1-\Phi s^{2}} d s .
$$

Let $s=-e^{i \theta}$ and $t=\cos \theta$; then using $T_{k}(\cos \theta)=\cos (k \theta)$, we have

$$
\begin{aligned}
& -\frac{\Phi^{2}}{2 \pi i} \oint_{\gamma} f(z)\left[1+s^{\prime}(z)\right] \frac{s^{5}(z)}{1-\Phi s^{2}(z)} d z=-\frac{\Phi^{2}}{2 \pi i} \oint_{|s|=1} f\left(-s-s^{-1}\right) \frac{s^{3}}{1-\Phi s^{2}} d s \\
& =\frac{\Phi^{2}}{2 \pi} \int_{-\pi}^{\pi} f(2 \cos \theta) \frac{e^{4 i \theta}}{1-\Phi e^{2 i \theta}} d \theta \\
& =\frac{\Phi^{2}}{2 \pi} \int_{-\pi}^{\pi} f(2 \cos \theta)\left[-\frac{\cos (2 \theta)}{\Phi}-\frac{1}{2 \Phi^{2}}+\frac{1-\Phi^{2}}{2 \Phi^{2}}\left(1+\Phi^{2}-2 \Phi \cos (2 \theta)\right)^{-1}\right] d \theta \\
& =\frac{\Phi^{2}}{\pi} \int_{-1}^{1} f(2 t)\left[-\frac{T_{2}(t)}{\Phi}-\frac{1}{2 \Phi^{2}}+\frac{1-\Phi^{2}}{2 \Phi^{2}}\left[1+\Phi^{2}-2 \Phi T_{2}(t)\right]^{-1}\right] \frac{1}{\sqrt{1-t^{2}}} d t \\
& =-\Phi \tau_{2}(f)-\frac{1}{2} \tau_{0}(f)+\frac{1-\Phi^{2}}{2 \pi} \int_{-1}^{1} f(2 t)\left[1+\Phi^{2}-2 \Phi T_{2}(t)\right]^{-1} \frac{1}{\sqrt{1-t^{2}}} d t .
\end{aligned}
$$

This equality together with (5.3) implies (1.5).

To calculate the integral

$$
-\frac{\Phi}{4 \pi^{2}} \oint_{\gamma_{1}} \oint_{\gamma_{2}} f\left(z_{1}\right) g\left(z_{2}\right) s_{1}^{\prime} s_{2}^{\prime} \frac{1}{\left(1-\Phi s_{1} s_{2}\right)^{2}} d z_{1} d z_{2}
$$

we will use the same procedure as above. For $\rho_{1}<\rho_{2}<1$, just as the definition of $\gamma^{\prime}$, we can define contours $\gamma_{j}^{\prime}, j=1,2$. A similar argument together with the Taylor expansion

$$
\left(1-\Phi s_{1} s_{2}\right)^{-2}=\sum_{k=0}^{\infty}(k+1)\left(\Phi s_{1} s_{2}\right)^{k}
$$

leads to

$$
\begin{aligned}
& -\oint_{\gamma_{1}} \oint_{\gamma_{2}} f\left(z_{1}\right) g\left(z_{2}\right) s_{1}^{\prime} s_{2}^{\prime} \frac{1}{\left(1-\Phi s_{1} s_{2}\right)^{2}} d z_{1} d z_{2} \\
& =\int_{-\pi}^{\pi} \int_{-\pi}^{\pi} f\left(2 \cos \theta_{1}\right) g\left(2 \cos \theta_{2}\right) \sum_{k=0}^{\infty}(k+1) \Phi^{k} e^{i(k+1)\left(\theta_{1}+\theta_{2}\right)} d \theta_{1} d \theta_{2} .
\end{aligned}
$$

From the definition of $\tau_{k}(f)$ and the bound $\left|\tau_{k}(f)\right| \leqslant C /(k(k-1))$ (see [4]), we get $(5.5)=\sum_{\ell=1}^{\infty} \ell \Phi^{\ell} \tau_{\ell}(f) \tau_{\ell}(g)$. This equation together with (5.4) leads to (1.6). Thus the proof of Theorem 1.1 is completed. 


\section{REFERENCES}

1. Anderson G. W., Zeitouni O. A CLT for a band matrix model. - Probab. Theory Related Fields, 2006, v. 134, № 2, p. 283-338.

2. Bai Z. D., Silverstein J. W. Spectral Analysis of Large Dimensional Random Matrices. Beijing: Science Press, 2006, 393 p.

3. Bai Z. D., Wang X. Y., Zhou W. CLT for linear spectral statistics of Wigner matrices. - Electron. J. Probab., 2009, v. 14, № 83, p. 2391-2417.

4. Bai Z.D., Yao J.F. On the convergence of the spectral empirical process of Wigner matrices. - Bernoulli, 2005, v. 11, № 6, p. 1059-1092.

5. Bai Z.D., Yin Y.Q. Necessary and sufficient conditions for almost sure convergence of the largest eigenvlues of a Wigner matrix. - Ann. Probab., 1988, v. 16, № 4, p. 1729-1741.

6. Diaconis P., Evans S. N. Linear functionals of eigenvalues of random matrices. Trans. Amer. Math. Soc., 2001, v. 353, № 7, p. 2615-2633.

7. Guhr T., Müller-Groeling A., Weidenmüller H. A. Random-matrix theories in quantum physics: common concepts. - Phys. Rep., 1998, v. 299, № 4-6, p. 189-425.

8. Johansson $K$. On fluctuations of eigenvalues of random Hermitian matrices. - Duke Math. J., 1998, v. 91, № 1, p. 151-204.

9. Jonsson D. Some limit theorems for the eigenvalues of a sample covariance matrix. J. Multivariate Anal., 1982, v. 12, p. 1-38.

10. Марченко В. А., Пастур Л. А. Распределение собственных значений в некоторых ансамблях случайных матриц. - Матем. сб., 1967, т. 72, № 4, с. 507-536.

11. Mehta M. L. Random matrices. Amsterdam: Elsevier, 2004, 688 p.

12. Shcherbina M. Central limit theorem for linear eigenvalue statistics of the Wigner and sample covariance random matrices. - J. Math. Phys. Anal. Geo., 20011, v. 7, № 2, p. 176-192.

13. Sinaǐ Y. A., Soshnikov A. Central limit theorem for traces of large random symmetric matrices with independent entries. - Bull. Braz. Math. Soc., 1998, v. 29, № 1, p. 1-24.

14. Pan G. M., Zhou W. Central limit theorem for signal-to-interference ratio of reduced rank linear receiver. - Ann. Appl. Probab., 2008, v. 18, № 3, p. 1232-1270.

15. Pandey A., Mehta M. L. Gaussian ensembles of random Hermitian matrices intermediate between orthogonal and unitary ones. - Comm. Math. Phys., 1983, v. 87, № 4, p. 449-468.

16. Lytova A., Pastur L. A. Central limit theorem for linear eigenvalues statistics of random matrices with independent entries. - Ann. Probab., 2009, v. 37, № 5, p. 17781840 .

Поступила в редакцию

21.VIII.2012

Исправленный вариант

29.VI.2013 